\title{
Molecular and Clinicopathologic Heterogeneity of Intracranial Tumors Mimicking Extraskeletal Myxoid Chondrosarcoma
}

Velz, Julia ; Agaimy, Abbas ; Frontzek, Karl ; Neidert, Marian Christoph ; Bozinov, Oliver ; Wagner, Ulrich ; Fritz, Christine ; Coras, Roland ; Hofer, Silvia ; Bode-Lesniewska, Beata ; Rushing, Elisabeth

\begin{abstract}
Primary intracranial neoplasms with features of extraskeletal myxoid chondrosarcomas (EMC) are extremely rare and poorly characterized tumors with only 12 cases described, the majority lacking molecular confirmation. There is an urgent need for the integration of molecular studies for correct subclassification of these tumors in order to predict clinical behavior, guide therapeutic decision-making, and provide novel targets for therapy. Clinical and pathologic data of 3 intracranial EMC-like myxoid neoplasms were retrospectively reviewed. In $2 / 3$ cases, immunohistochemistry showed loss of nuclear SWI/SNF-related matrix-associated actin-dependent regulator of chromatin subfamily B member 1 (SMARCB1; integrase interactor 1 [INI1]) staining accompanied by monosomy of chromosome 22q (fluorescence in situ hybridization [FISH]). These 2 cases had no evidence of any fusion products by next generation sequencing (NGS). The third case had intact SMARCB1 expression and showed instead a rearrangement of the EWSR1 gene detected by FISH, with an EWSR1-CREB1 gene fusion on NGS. None of the cases showed rearrangement of the NR4A3 gene, neither by FISH nor by NGS. This small case series highlights the molecular heterogeneity of intracranial neoplasms in the morphologic spectrum of EMC. Distinct molecular alterations found in tumors with morphologic features of EMC encompass SMARCB1(INI1) loss and EWSR1-CREB gene fusions. None of the cases showed rearrangements of NR4A3 genes, suggesting they are distinct from conventional EMC.
\end{abstract}

DOI: https://doi.org/10.1093/jnen/nly050

Posted at the Zurich Open Repository and Archive, University of Zurich

ZORA URL: https://doi.org/10.5167/uzh-152107

Journal Article

Published Version

Originally published at:

Velz, Julia; Agaimy, Abbas; Frontzek, Karl; Neidert, Marian Christoph; Bozinov, Oliver; Wagner, Ulrich; Fritz, Christine; Coras, Roland; Hofer, Silvia; Bode-Lesniewska, Beata; Rushing, Elisabeth (2018). Molecular and Clinicopathologic Heterogeneity of Intracranial Tumors Mimicking Extraskeletal Myxoid Chondrosarcoma. Journal of Neuropathology and Experimental Neurology, 77(8):727-735.

DOI: https://doi.org/10.1093/jnen/nly050 


\title{
Molecular and Clinicopathologic Heterogeneity of Intracranial Tumors Mimicking Extraskeletal Myxoid Chondrosarcoma
}

\author{
Julia Velz, MD, Abbas Agaimy, MD, Karl Frontzek, MD, Marian Christoph Neidert, MD, \\ Oliver Bozinov, MD, Ulrich Wagner, PhD, Christine Fritz, MSc, Roland Coras, MD, \\ Silvia Hofer, MD, Beata Bode-Lesniewska, MD, and Elisabeth Rushing, MD
}

\begin{abstract}
Primary intracranial neoplasms with features of extraskeletal myxoid chondrosarcomas (EMC) are extremely rare and poorly characterized tumors with only $\sim 12$ cases described, the majority lacking molecular confirmation. There is an urgent need for the integration of molecular studies for correct subclassification of these tumors in order to predict clinical behavior, guide therapeutic decision-making, and provide novel targets for therapy. Clinical and pathologic data of 3 intracranial EMC-like myxoid neoplasms were retrospectively reviewed. In $2 / 3$ cases, immunohistochemistry showed loss of nuclear SWI/SNF-related matrix-associated actindependent regulator of chromatin subfamily $\mathrm{B}$ member 1 (SMARCB1; integrase interactor 1 [INI1]) staining accompanied by monosomy of chromosome $22 \mathrm{q}$ (fluorescence in situ hybridization [FISH]). These 2 cases had no evidence of any fusion products by next generation sequencing (NGS). The third case had intact SMARCB1 expression and showed instead a rearrangement of the EWSRI gene detected by FISH, with an EWSRI-CREBI gene fusion on NGS. None of the cases showed rearrangement of the NR4A3 gene, neither by FISH nor by NGS. This small case series highlights the molecular heterogeneity of intracranial neoplasms in the morphologic spectrum of EMC. Distinct molecular alterations found in tumors with morphologic features of EMC encompass SMARCB1(INI1) loss and EWSRI-CREB gene fusions. None of the cases showed rearrangements of $N R 4 A 3$ genes, suggesting they are distinct from conventional EMC.
\end{abstract}

From the Department of Neurosurgery, Clinical Neuroscience Center, University Hospital Zurich, Zurich, Switzerland (JV, MCN, OB); University of Zurich, Zurich, Switzerland (JV, KF, MCN, OB, UW, CF, BB-L, ER); Department of Pathology, University Hospital Erlangen, Erlangen, Germany (AA); Department of Neuropathology, Clinical Neuroscience Center (KF, ER); Institute of Pathology and Molecular Pathology, University Hospital Zurich, Zurich, Switzerland (UW, CF, BB-L); Department of Neuropathology, University Hospital Erlangen, Erlangen, Germany (RC); and Department of Oncology, Hospital Lucerne, Lucerne, Switzerland $(\mathrm{SH})$

Send correspondence to: Elisabeth J. Rushing, MD, Department of Neuropathology, University Hospital Zurich, Schmelzbergstrasse 12, CH-8091 Zurich, Switzerland; E-mail: elisabethjane.rushing@usz.ch

Beata Bode-Lesniewska and Elisabeth Rushing are both senior authors.

The authors have no duality or conflicts of interest to declare.
Key Words: Cartilaginous tumors, EWSR1, Intracranial extraskeletal myxoid chondrosarcoma, SMARCB1 (INI1) loss.

\section{INTRODUCTION}

Recent advances in understanding the molecular background of mesenchymal neoplasms with evident or presumed cartilaginous differentiation have led to extensive revision in the classification. Historically, conventional, mesenchymal and extraskeletal myxoid chondrosarcomas (EMC) have been distinguished, with the conventional type being by far the most common in both extracranial and cranial locations (1-3). Intracranial cartilaginous tumors are rare, accounting for $<0.16 \%$ of all space-occupying brain tumors (4). The distribution of reported primary intracranial EMC-like neoplasms is highly variable with lesions described in the pineal region, frontal, temporal and parietal cortex, the falx, fourth ventricle and the cerebellum (1, 5-13) (Table 1). However, the lack of rigorous diagnostic criteria and the use of ambiguous terminology have resulted in misclassification of cartilaginous tumors in the past. For example, higher-grade conventional chondrosarcoma of the skull containing a myxoid rather than hyaline extracellular matrix (as is typical for low-grade conventional chondrosarcoma) has been misclassified as "myxoid chondrosarcoma". Nosologic imprecision has led to confusion with the histogenetically ambiguous but biologically distinct entity of EMC, which has eroded the significance of previous publications on this entity.

The current classification of mesenchymal tumors continues to evolve by incorporating more genetic information in the diagnostic algorithm (14). Among the chondrogenic tumors, conventional chondrosarcomas are characterized by mutations in the IDHI/IDH2 genes, while mesenchymal chondrosarcomas and EMC belong to translocation associated sarcomas involving the HEYI-NCOA2 and NR4A3 genes, respectively (15).

None of the older publications contains information on the genetic background of the reported tumors. In fact, only very few recent papers address the underlying genetic mechanisms of intracranial myxoid chondrogenic neoplasms, which 
TABLE 1. Cases of Intracranial Extraskeletal Myxoid Chondrosarcoma Described in the Literature

\begin{tabular}{|c|c|c|c|c|}
\hline Author & Age/Sex & Location/Attachment & Treatment & Outcome \\
\hline Scott (1976) (5) & $39 / \mathrm{M}$ & 4th ventricle, choroid plexus & Surgery & 13 days, died (ventriculitis) \\
\hline Smith (1981) (12) & $12 / \mathrm{M}$ & $\begin{array}{l}\text { Left cerebellar hemisphere and foramen } \\
\text { magnum (dura) }\end{array}$ & Surgery & 13 months, alive, free of disease \\
\hline Salcman (1992) (28) & $28 / \mathrm{F}$ & Left parafalcine and dura of falx & Surgery and brachytherapy & 20 months, alive, local recurrence \\
\hline Sato (1993) (29) & $43 / \mathrm{F}$ & Pineal gland and dura & $\begin{array}{l}\text { Surgery, radiotherapy } \\
\text { and chemotherapy }\end{array}$ & 3 years, died (tumor progress) \\
\hline Gonzalez-Lois (2002) (1) & $17 / \mathrm{F}$ & Fronto-temporal (dura and hemisphere) & Surgery and radiotherapy & 19 months, alive, local recurrence \\
\hline Chaskis (2002) (6) & $69 / \mathrm{M}$ & Parietal, Cortex left & Surgery and radiotherapy & $\begin{array}{l}1 \text { months died with septic shock } \\
\text { d/t diverticulitis }\end{array}$ \\
\hline $\operatorname{Im}(2003)(7)$ & $43 / \mathrm{M}$ & Parietal, Cortex left & Surgery and radiotherapy & 3 years alive, no recurrence \\
\hline Sorimachi (2008) (13) & $37 / \mathrm{F}$ & $\begin{array}{l}\text { Pineal gland (no dura or plexus } \\
\text { choroideus attachment) }\end{array}$ & $\begin{array}{l}\text { 1st surgery } \\
\text { 2nd surgery }\end{array}$ & $\begin{array}{l}\text { 1st: } 13 \text { months recurrence } \\
\text { 2nd: } 7 \text { months alive, no tumor } \\
\text { recurrence }\end{array}$ \\
\hline O'Brien (2008) (8) & $26 / \mathrm{F}$ & Left cerebellopontine angle & $\begin{array}{l}\text { 1st surgery } \\
\text { 2nd surgery } \\
\text { proton therapy }\end{array}$ & $\begin{array}{l}1 \text { year after surgery no evidence } \\
\text { of any tumor enlargement }\end{array}$ \\
\hline Arpino (2011) (30) & $54 / \mathrm{F}$ & Sellar/left parasellar mass & Surgery & ND \\
\hline Park (2012) (9) & $21 / \mathrm{F}$ & Left lateral ventricle/choroid plexus & Surgery and radiotherapy & 6 months, alive \\
\hline Dulou (2012) (10) & $70 / \mathrm{m}$ & Left frontal Lobe & $\begin{array}{l}\text { Radiotherapy, surgery } \\
\text { and chemotherapy }\end{array}$ & 10 months, died \\
\hline Lee $(2015)(11)$ & $67 / \mathrm{m}$ & Left temporal Lobe & Radiotherapy and surgery & ND \\
\hline Described Case No.1 & $58 / \mathrm{F}$ & Pineal Gland & Surgery and Radiotherapy & $\mathrm{ND}$ \\
\hline Described Case No. 2 & $30 / \mathrm{F}$ & Left cerebellopontine angle & Surgery and radiotherapy & 4 months, alive \\
\hline Described Case No. 3 & $17 / \mathrm{M}$ & Left cerebellopontine angle & Surgery and radiotherapy & 3 years alive; tumor recurrence \\
\hline
\end{tabular}

microscopically correspond to the rather broad category of EMC. Interestingly, Kao and Bale report translocations of the EWSR1-CREB-family of genes translocations in 7 cases (4 cases EWSR1-CREB1, 2 cases EWSRI-CREM, and 1 case EWSR1-ATF1) $(16,17)$ (Table 2). These translocations have previously been reported in rare, mostly mesenchymal tumors of divergent clinical potential and heterogeneous histogenetic origin/line of differentiation. These entities encompass clear cell sarcoma, clear cell sarcoma-like tumor of the gastrointestinal tract (CCSLTGT) (synonym: malignant gastrointestinal neuroectodermal tumor), angiomatoid fibrous histiocytoma (AFH), and primary pulmonary myxoid sarcoma (PPMS) in addition to epithelial neoplasms such as hyalinizing clear cell carcinoma (HCCC) of the salivary gland and sclerosing clear cell odontogenic carcinoma (18). It seems that there is a peculiar subgroup of intracranial myxoid tumors, morphologically mimicking EMC, which harbors this translocation as well, broadening the spectrum of tumors linked to the EWSR1$C R E B$-family of translocation genes. Integrase interactor 1 (INI1) status was also investigated because rare extraskeletal tumors resembling EMC show loss of this marker (19).

In order to further elucidate the molecular background of the intracranial myxoid neoplasms previously classified as EMC, we studied 3 such tumors, collecting available clinical information and applying conventional, immunohistochemical and molecular (fluorescence in situ hybridization [FISH], next generation sequencing [NGS]) methods.

\section{MATERIALS AND METHODS}

All procedures performed in studies involving human participants were in accordance with the ethical standards of the institutional and/or national research committee and with the 1964 Helsinki declaration and its later amendments or comparable ethical standards.

Three cases of "intracranial EMC" were retrieved from the pathology files of our respective institutions. Hematoxylin and eosin (H\&E) sections and immunohistochemical stains performed at the time of diagnosis were reviewed. Clinical and follow-up data were obtained from the clinical database. FISH and NGS technology were used to investigate molecular alterations.

This study was approved by our institutional review board (the Cantonal Ethics Committee; KEK_ZH 20130430).

\section{Histology and Immunohistochemistry}

Tumor tissue samples were fixed in buffered $4 \%$ formalin and embedded in paraffin. The $2-\mu \mathrm{m}$-thick sections were stained with H\&E according to standard procedures. Immunohistochemistry using the SWI/SNF-related matrix-associated actin-dependent regulator of chromatin subfamily B member 1 (SMARCB1) (INI1) antibodies (clone MRQ-27, Zytomed [dilution 1:50] and clone BAF47, BD Biosciences [dilution 1:300]), (Franklin Lakes, NJ) was performed on 2- $\mu$ m-thick paraffin sections, using the Ventana Benchmark XT 
TABLE 2. Recently Described Intracranial Myxoid Chondrogenic Neoplasms (iMCN) With Genetic Information

\begin{tabular}{|c|c|c|c|c|c|}
\hline \multirow[t]{2}{*}{ Author } & \multirow[t]{2}{*}{ Age/Sex } & \multirow[t]{2}{*}{ Location/Attachment } & \multicolumn{2}{|c|}{ Immunohistochemistry } & \multirow[t]{2}{*}{ Fusion Type } \\
\hline & & & EMA & Desmin & \\
\hline Kao et al (16) & $15 / \mathrm{F}$ & Meninges & $\mathrm{F}+$ & $\mathrm{D}+$ & ESWR1-CREM \\
\hline Kao et al (16) & 20/M & Brain (frontal) & $\mathrm{F}+$ & $\mathrm{F}+$ & ESWR1-CREB1 \\
\hline Kao et al (16) & $12 / \mathrm{M}$ & Brain (frontal) & $\mathrm{F}+$ & $\mathrm{F}+$ & ESWR1-ATF1 \\
\hline Bale et al (17) & $12 / \mathrm{M}$ & Posterior fossa/cerebellar, abutting dura & $\mathrm{F}+$ & $\mathrm{D}+$ & ESWR1-CREB1 \\
\hline Bale et al (17) & $18 / \mathrm{M}$ & $\begin{array}{l}\text { Midline/right frontal (falx area), surrounded } \\
\text { by a thin rim of brain parenchyma }\end{array}$ & $\mathrm{F}+$ & $\mathrm{D}+$ & ESWR1-CREM \\
\hline
\end{tabular}

automated staining system (Ventana Medical Systems, Tucson, AZ).

\section{Fluorescence In Situ Hybridization}

The FISH studies were performed on formalin-fixed, paraffin-embedded, 2- $\mu$ m-thick tissue sections. Dual color break-apart FISH detecting translocations involving the EWSR1 (22q11) and NR4A3 (9q22.33-q31.1) genes was performed using commercially available probes (Vysis, Abbott AG, Baar, Switzerland and ZytoVision GmbH, Bremerhaven, Germany, respectively). Fluorescence staining was visualized with an Olympus BX61 microscope (Olympus, Volketswil, Switzerland) equipped with DAPI, SpectrumGreen and SpectrumOrange filters. Images were acquired with a CCD camera and processed with AnalySIS imaging software (Soft Imagining System, Munster, Germany). For the assessment of EWSR 1 and NR4A3 gene rearrangements, at least 50 nonoverlapping tumor nuclei were analyzed. If the sample contained at least $25 \%$ of split red and green signals, the tumor was regarded as translocation-positive. The signal was considered as split, when red and green fluorescent spots were separated by at least twice the distance occupied by a single probe.

To detect copy number alterations of the SMARCBI (INI1) gene locus, FISH was performed using the ZytoLight SPEC SMARCB1/22q12 Dual Color Probe, which is a mixture of a green fluorochrome direct labeled SPEC SMARCB1 probe hybridizing to the human $S M A R C B 1$ gene in the chromosomal region $22 \mathrm{q} 11.23$ and an orange fluorochrome direct labeled SPEC 22q12 probe as supplied by the manufacturer (ZytoVision $\mathrm{GmbH}$ ). Absence of 1 or both of $S M A R C B 1$ green signals but intact 22q12 orange signals is reported as monoallelic and biallelic deletion of $S M A R C B 1$, respectively. Absence of 1 orange and 1 green signal is reported as monosomy $22 \mathrm{q}$ or at least part of it spanning both $S M A R C B 1$ gene locus and $22 \mathrm{q}$ region.

\section{Next Generation Sequencing}

Ribonucleic acid (RNA) was extracted using the Maxwell 16 LEV RNA FFPE Purification Kit (Promega Corporation, Madison, WI) according to the manufacturer's instructions.
Libraries were prepared using Anchored Multiplex PCR with the commercially available Archer FusionPlex Sarcoma Panel (ArcherDx, Boulder, CO), which simultaneously identifies fusions of 26 genes associated with soft tissue sarcomas. The RNA input was as follows: Case 1: $250 \mathrm{ng}$, Case 2: $80 \mathrm{ng}$, and Case 3: 250 ng. Briefly, complementary DNA was synthesized using random primers. After synthesis of the first strand, $1 \mu 1$ of the reaction was run in a PreSeq assay (qPCR) to ensure sufficient RNA quality. Synthesis of the second cDNA strand was followed by an end repair step and libraries were cleaned using Agencourt AMPure XP beads (Beckman Coulter, Brea, CA). Samples were adenylated with a first ligation step and cleaned again with AMPure beads. In a second ligation step a molecular barcode adapter was incorporated to the libraries, which contains a random 8-mer used to differentiate individual nucleic acid molecules in the samples before any amplification occurs. After cleanup with ligation cleanup beads (ArcherDx), libraries were amplified in a first PCR reaction using gene-specific primers (GSP1), cleaned with AMPure beads, followed by a second PCR reaction with gene-specific primers (GSP2) to increase amplicon specificity perform target enrichment and add an index for multiplexing. Libraries were then quantified using qPCR (KAPA Biosystems, South San Francisco, CA), normalized and processed for sequencing. Samples were sequenced on the MiSeq platform (Illumina, San Diego, CA) with a minimum of 2 million reads per sample.

\section{Sequencing Data Analysis}

The resulting fast-files were analyzed using the standard RNA fusion workflow as implemented in the Archer Analysis Suite 5.1.3. Briefly, after adapter trimming, reads were deduplicated using the random 8-mer molecular barcode. Reads were then mapped to control and target regions and finally to the human genome (hg19). Chimeric reads aligning to 2 separate genes with little or no overlap were considered fusion candidates. Reads with identical breakpoints in the fused genes were used to establish a consensus sequence. Subsequently, reads were then mapped back to this consensus. 

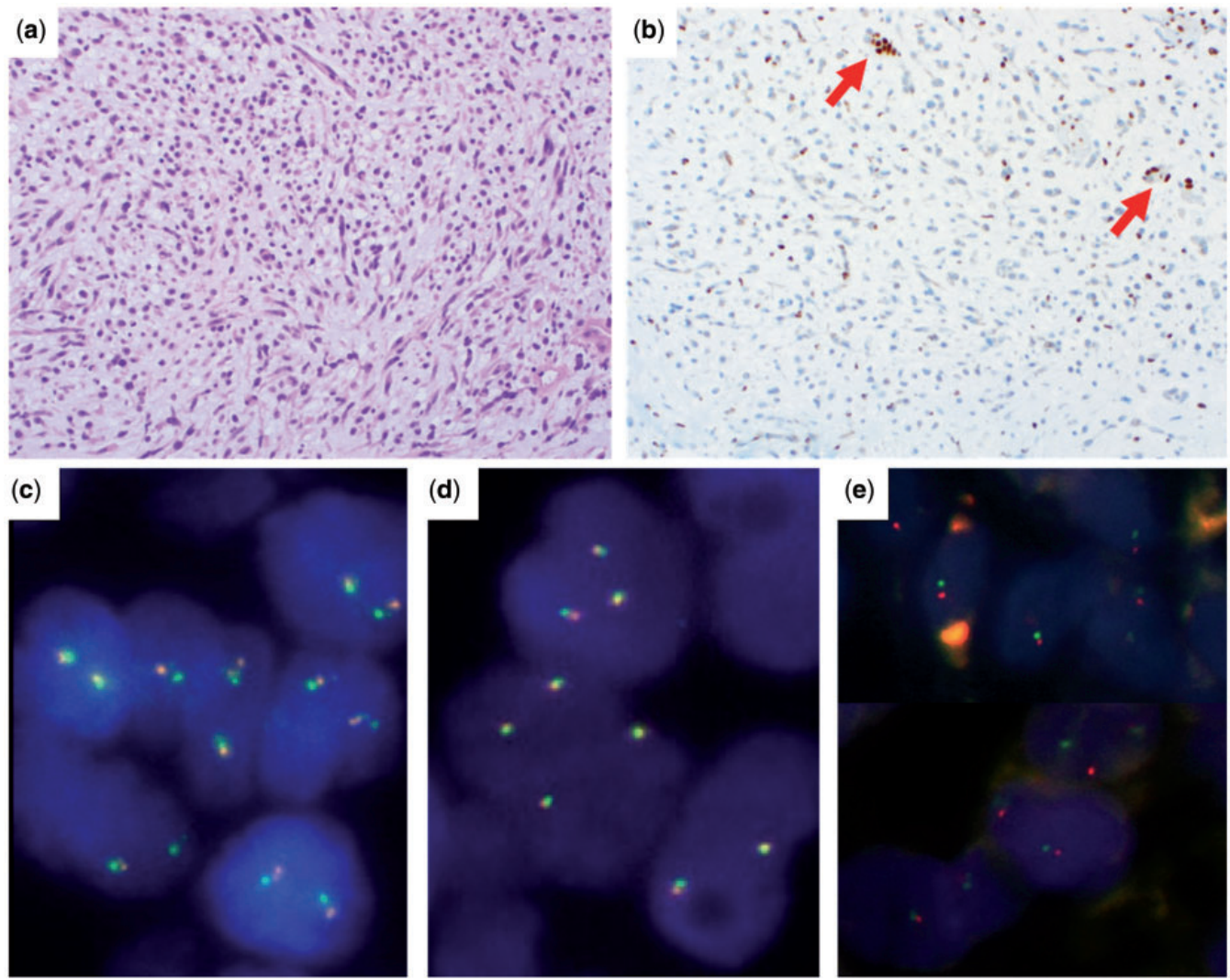

FIGURE 1. (Case 1) Tumor of the pineal region of a 58-year-old woman. (A) H\&E stain, original magnification: $\times 200$. (B) Loss of nuclear expression of INI1 protein in the tumor cells in contrast to non-neoplastic nuclei of the endothelial cells (arrows), original magnification: $\times 200$ ). (C, D) Interphase FISH demonstrates lack of rearrangement of the EWSR1 and NR4A3 genes, respectively. The tumor nuclei contain exclusively fused green and orange signals. (E) FISH demonstrates monosomy for the $22 \mathrm{q}$ gene locus with single INI1/SMARCB1 signal and single centromere signal.

\section{RESULTS}

\section{Clinical Features}

Case 1 (Fig. 1). A 58-year-old female patient was referred to the Neurosurgery Department of the University Hospital of Zurich after an episode of vertigo and visual disturbances accompanied by a change in her usual headache pattern. MRI revealed a space-occupying lesion in the pineal region. The patient underwent a midline suboccipital osteoplastic craniotomy with resection of the tumor. Histopathologic examination revealed an intracranial neoplasm consistent with EMC. The perioperative period was uneventful. Postoperative resection control (MRI) showed radiologic evidence of a residual mass and the patient was referred for stereotactic irradiation of the residual tumor. A whole-body CT and/or PET to exclude further tumor sites was not performed. The patient was postoperatively lost to follow-up.

Case 2 (Fig. 2). A 30-year-old woman was referred to the neurosurgery department of an external hospital with a newly diagnosed mass occupying the left cerebellopontine angle with extrameatal and intrameatal components and signs of brainstem compression. The patient had presented with new onset of vertigo of several weeks duration. ENT examination revealed slight hearing loss and vestibular dysfunction on the left side. The suspected initial diagnosis was vestibular schwannoma. The lesion was removed using microsurgical techniques via suboccipito-lateral trepanation. Intraoperatively, the cochlear nerve was seen attached to and traversing the entire mass. The part of the lesion associated with the fascial nerve could not be removed completely. Postoperative PET-CT showed no evidence of metastasis or another extracranial primary tumor. Adjuvant radiotherapy was given at a total dose of $63 \mathrm{~Gy}$. At last follow-up, 4 months after surgery, the patient was alive and without evidence of disease after completion of the radiation therapy.

Case 3 (Fig. 3). A 17-year-old boy was referred to the Children's Health Centre of an external hospital with a newly diagnosed lesion with perifocal edema occupying the left cerebellopontine angle in December 2014 (Fig. 4). The patient, who suffered from migraine, noted a change of his usual headache pattern over several weeks in association with nausea. Upon admission, he showed lower cranial nerve deficits (VII, IX, X) without explicit cerebellar signs. A left retromastoid suboccipital craniotomy was performed with removal of the tumor within the visible boundaries. The perioperative period was uneventful. The postoperative resection control (MRI) showed no radiologic signs of any residual mass (Fig. 4). The histopathology was reevaluated several times, including at multiple international brain tumor reference centers. Initially, 

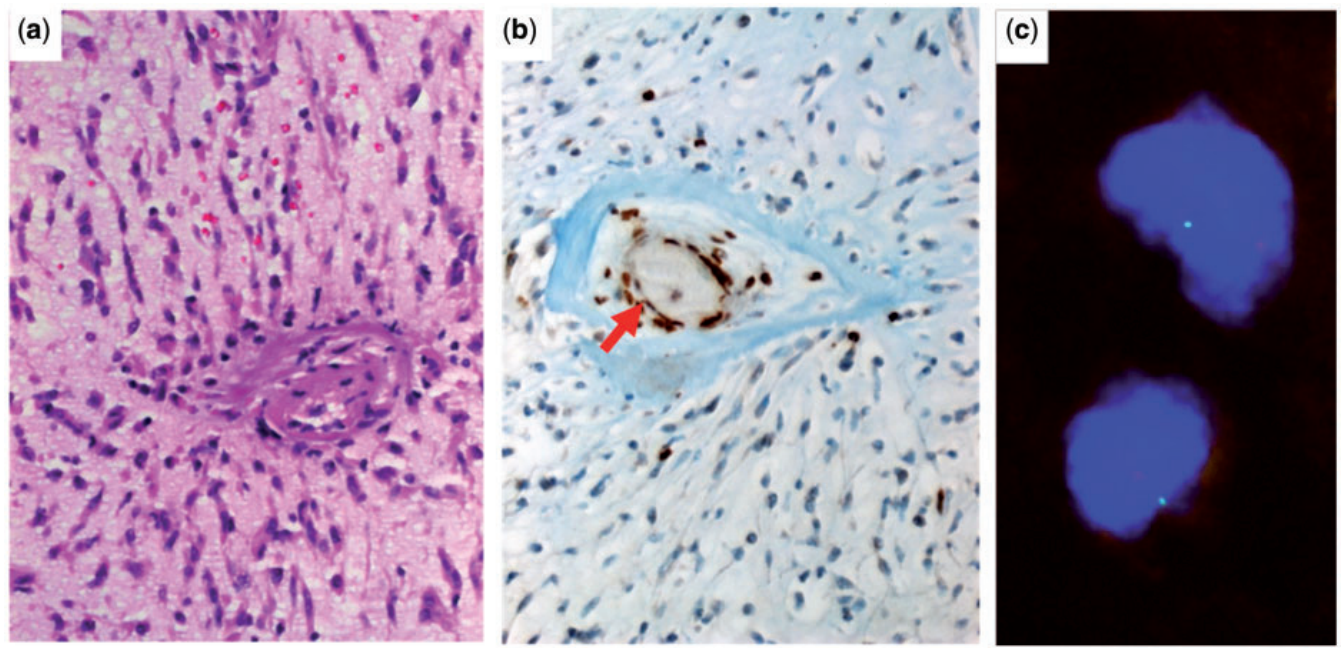

FIGURE 2. (Case 2) Intracranial tumor in the left cerebellopontine angle of the 30-year-old woman. (A) H\&E stain, original magnification: $\times 200$. (B) Loss of nuclear expression of INI1 protein in the tumor cells in contrast to non-neoplastic nuclei of the blood vessels (arrow), original magnification: $\times 200$. (C) FISH demonstrates monosomy for the INI1/SMARCB1 gene/22q: The nuclei of the tumor cell contain one signal each. The FISH showed no rearrangement of the EWSR1 and N4RA3 genes (not shown).
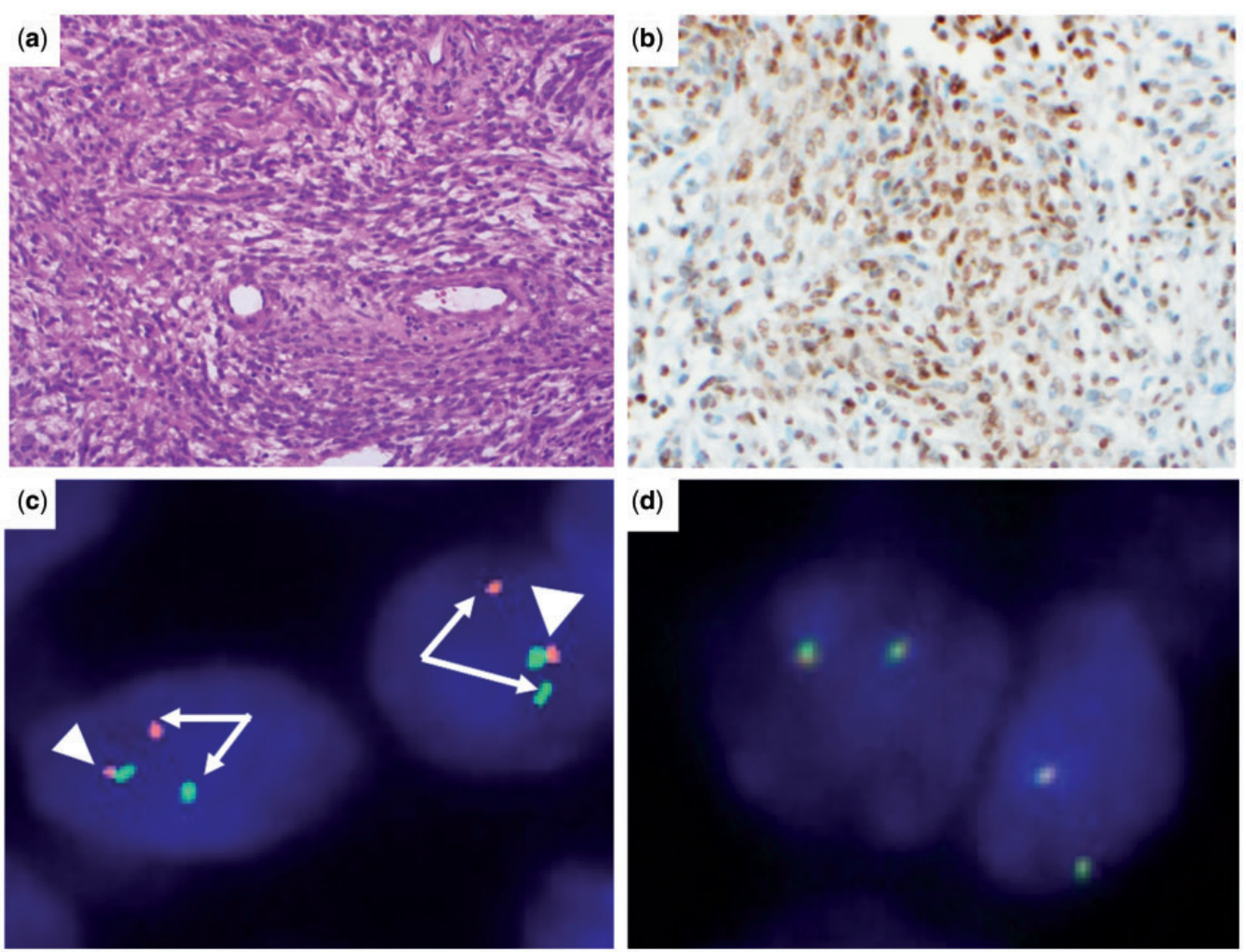

(d)

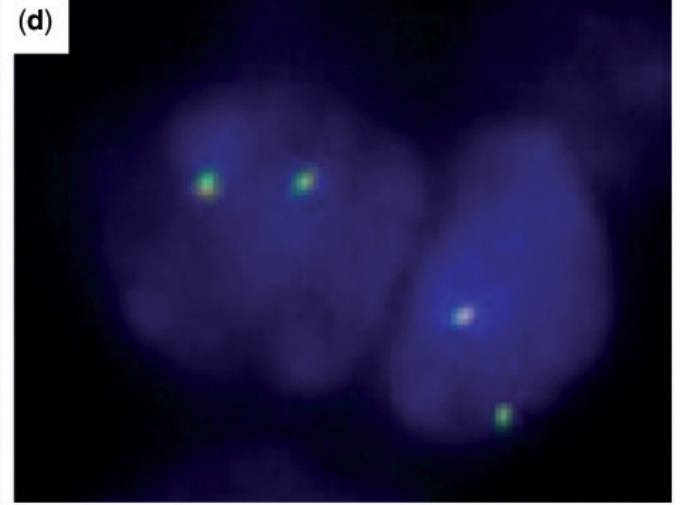

FIGURE 3. (Case 3) Infratentorial tumor of the 17-year-old boy. (A) H\&E stain, original magnification: $\times 200$. (B) Retained nuclear expression of INI1 protein, original magnification: $\times 200$. (C) Interphase FISH demonstrating the rearrangement of the EWSR1 gene. One fused gene signal (arrowhead) is accompanied by split green and orange signals (arrows) in the tumor nuclei. (D) Interphase FISH demonstrates the lack of rearrangement of the NR4A3 gene. The tumor nuclei contain exclusively fused green and orange signals. FISH analysis shows normal disomic pattern for the INI1/SMARCB1 gene (not shown). 

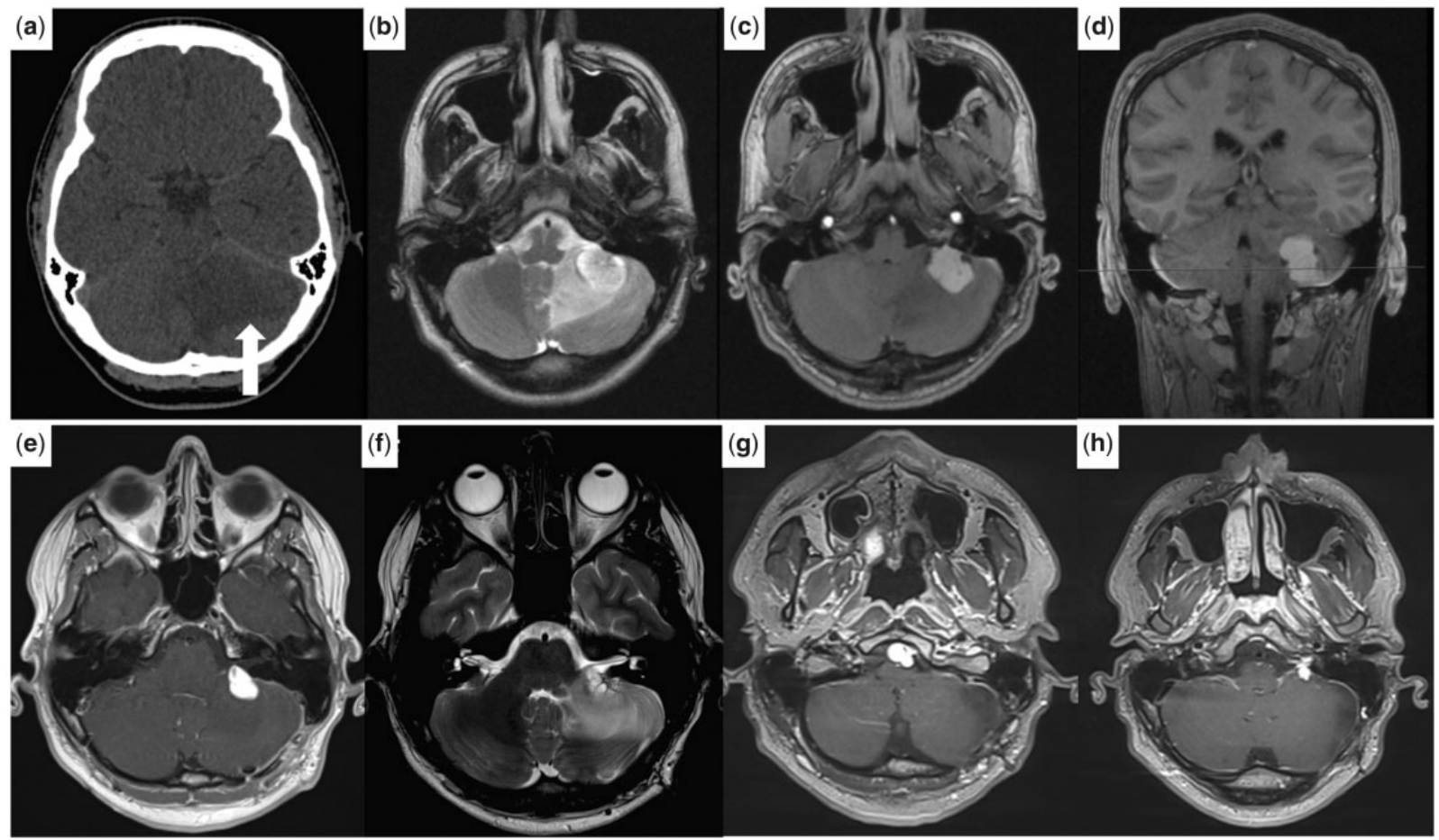

FIGURE 4. (Case 3) Soft tissue window of preoperative axial CT scan (A). (B) Axial section of preoperative T2-weighted MRI shows a newly diagnosed mass with perifocal edema occupying the left cerebellopontine angle. Axial (C) and coronal (D) sections of preoperative T1-weighted and contrast-enhanced MRI (December 2014). Axial and contrast-enhanced section of T1-weighted (E) and axial T2-weighted MRI (F) in August 2015 reveal recurrence of the infratentorial lesion; recraniotomy was performed to remove the recurrent tumor in September 2015. During follow-up in September 2016, axial sections of T1-weighted and contrast-enhanced $\operatorname{MRI}(\mathbf{G}, \mathbf{H})$ displayed 6 new extracerebral nodules in the basal cistern (only 2 lesion sites are shown, G, H).

a meningioma or schwannoma was discussed. Radiologic follow-up examination was performed 4 and 8 months after surgery, which showed a recurrence of the infratentorial lesion. In September 2015, the patient was referred to another external hospital, where recraniotomy with a suboccipital approach was undertaken to remove recurrent tumor from the left cerebellopontine angle. Again, there was uncertainty regarding the histopathologic diagnosis. Follow-up imaging 3 and 6 months after the second surgery did not show any evidence of recurrent tumor. In March 2016, the patient noted increased severity of headaches and vertigo. cMRI in August 2016 showed 6 new extracerebral nodules in the basal cistern (including 1 lesion in the region of previous surgery). In September 2016, the histologic samples were sent in consultation to the Neuropathology Department of the University of Zürich, where histopathologic examination revealed intracranial EMC. Rearrangement of the EWSRl gene was demonstrated by FISH analysis (Fig. 3C) (NR4A3 gene FISH and NGS were not available at that time). At last follow-up, 3 years after initial diagnosis, the patient was still alive and MRI showed recurrence of the tumor.

\section{Pathologic Findings \\ Microscopic Examination}

Case 1 (Fig. 1A). The tumor tissue consisted of variably dense cords of small to middle sized oval to spindled cells embedded in a prominent myxoid matrix. Focal areas with dense fibrotic material or more pronounced chondrohyaline matrix were observed. Mitotic figures were rare. No necrosis was found.

Case 2 (Fig. 2A). Histologic examination showed moderately atypical plump elongated to rounded epithelioid cells with vesicular chromatin. There were occasional small inconspicuous nucleoli and pale-eosinophilic cytoplasm with frequent plasmacytoid cell features resulting from paranuclear condensation of the cytoplasm. Foci of spindled and binucleated cells were seen as well. The neoplastic cells were arranged into reticular cords and trabeculae within a prominent myxoid fibrovascular stroma with occasional chondromyxoid features. Mitotic activity was in the range of 4 mitoses/10 high-power fields. The tumor was closely associated with nerve fibers, with areas of frank intraneural invasion.

Case 3 (Fig. 3A). Microscopic examination revealed cords and communicating reticular/pseudoacinar aggregates of monomorphic, round to oval tumor cells, embedded in a myxoid stroma, and imparting a lacelike appearance. Mitotic figures or necrosis was not observed; however, occasional apoptotic bodies were seen.

\section{Immunohistochemistry}

Case 1. Immunohistochemistry showed negativity for AE1/AE3, EMA, S100, CD34, p63, MUC4, CD117, SMA, 
TABLE 3. Immunohistochemical and Molecular Analyses

\begin{tabular}{lllllll}
\hline Case No. & Age/Sex & INI1 IHC & INI1 FISH & EWS FISH & NR4A3 FISH & FusionPlex Sarcoma Panel (Archer) \\
\hline 1 & $58 / \mathrm{F}$ & Loss & Monosomy & Negative & Negative & Negative \\
2 & $30 / \mathrm{F}$ & Loss & Monosomy & Negative & Negative & Negative \\
3 & 17/M & Nuclear & Disomy & Positive & Negative & EWSR1-CREB1 \\
\hline \multicolumn{7}{l}{ F, female; M, male. } \\
\hline
\end{tabular}

desmin, brachyury, and synaptophysin. There was loss of nuclear expression of SMARCB1 (Fig. 1B) in the tumor cells in contrast to non-neoplastic stromal or vascular cells in the background. The proliferation index (Ki67) was $<5 \%$.

Case 2. Immunohistochemistry showed consistent expression of EMA and smooth muscle actin. In addition, there was weak to moderate nuclear reactivity for ERG as well as scattered p63-positive cells. A panel of immunohistochemical stains was performed including S100, SOX10, desmin, MUC4, CD34, STAT6, SSTR2A, AE1/AE3, CD31, CD30, D2-40, ALK, Brachyury, and CD117; all were negative. The neoplastic cells showed complete loss of SMARCB1/INI1 (Fig. 2B), with retained expression in normal stromal cells. The proliferation fraction (Ki67) was $10 \%$.

Case 3. The tumor cells were negative for pancytokeratin, S100 protein and EMA, claudin-1, MUC4, and synaptophysin. Nuclear expression of INI1 protein was retained in the tumor cell nuclei (Fig. 3B). The Ki67 proliferation index was $10 \%$.

\section{FISH Analysis}

The results of the FISH analyses of all cases are summarized in Table 3. The FISH analysis of the EWSRI gene showed no rearrangement in the tumor of the Patients 1 and 2, while the FISH probe signals were split in the tumor of Patient 3, indicating a translocation involving this gene (Figs. 1C, 3C). None of the tumors showed rearrangement of the NR4A3 gene in the FISH analysis (Figs. 1D, 3D).

The SMARCB1/22q12 FISH analysis showed heterozygous deletion in the tumor cell nuclei of Patients 1 and 2 frequently associated with loss of the $22 \mathrm{q}$ centromere signals indicating monosomy at $22 \mathrm{q}$ locus, while Patient 3 had 2 SMARCBl gene signals per nucleus (Figs. 1E, 2C).

\section{Next Generation Sequencing}

NGS using the Archer FusionPlex Sarcoma Panel did not detect any gene fusions of the 26 genes studied in the tumor tissue of the Patients 1 and 2. In the tumor tissue of the $\mathrm{Pa}$ tient 3 an EWSR 1-CREB1 gene fusion was identified.

\section{DISCUSSION}

Intracranial EMC is exceptionally rare with only approximately a dozen cases reported since its first description in $1972(3,13)$ (Table 1$)$. Due to its rarity, the clinicopathologic and molecular characteristics of this neoplasm have not been sufficiently analyzed. High rates of recurrence in patients with intracranial EMC undergoing radical excision and adjuvant radiotherapy have been described in previous reports (1, 13). Treatment standard consists of radical excision to achieve total removal of the tumor $(1,2,8)$. Adjuvant therapies, including radiotherapy and proton beam treatment, have been found to improve patient outcomes for this rare cancer (Table 1). Nevertheless, there is an urgent need to identify molecular markers that can reliably predict clinical behavior, guide therapeutic decision-making and that can serve as novel targets for therapy. The field of sarcoma classification has entered a new era with the introduction of paradigms based on molecular alterations. Approximately one-third of all soft tissue tumors harbor recurrent (mainly balanced) chromosomal translocations, usually encoding aberrant chimeric transcription factors. Pathognomonic chromosomal translocations may even define specific sarcoma entities and can be used as highly specific prognostic markers in the appropriate clinical and pathologic context. However, it has become apparent that some gene fusions are not necessarily specific for a given histologic subtype but are shared among various sarcoma (or even carcinoma) types otherwise unrelated clinically or immunophenotypically. EWSR1, encoding the RNA-binding protein ESWR1, a member of the TET family of transcription factors, is a prototypical "promiscuous gene" with a propensity for fusing with a host of different genes. In fact, rearrangement of ESWR1 have been found in a variety of mesenchymal and other neoplasms including the Ewing family of tumors (18).

\section{ESWR1-NR4AR Fusion}

The rearrangement of the NRAA3 gene is, according to the current WHO classification, the defining molecular feature of EMC (14). In approximately two-thirds of cases of EMC, a chromosomal reciprocal translocation of $\mathrm{t}(9$; 22)(q22; q12) has been identified, which corresponds to the EWSR1/NR4A3 gene fusion (20). NR4A3 gene fusions to other gene partners including TATA binding protein-associated factor 15 (TAF15), transcription factor 12 (TCF12), and TRK-fused gene (TFG) have been described in a smaller proportion of cases and correlate with rhabdoid phenotype, high-grade morphology and a more aggressive outcome in EMC (20). None of the cases described in the current study showed the classical rearrangement of the EMC, involving the NR4A3 gene. 


\section{ESWR1 Fusion With CREB Family Transcription Factors}

Gene fusions of EWSRl with members of the cAMP response element binding protein (CREB), including $C R E B 1$ and $A T F 1$, have been identified in 5 histopathologically diverse neoplasms: AFH, conventional clear cell sarcoma, CCSLTGT, HCCC of the salivary gland, and PPMS (18, 21-24).

EWSR1-ATF 1 and/or EWSR1-CREB1 are examples of gene fusions that are not restricted to a specific histomorphologic pattern. The histologic overlap and nonspecific immunoprofile of different tumor types have led to the discussion of a single pathologic entity characterized by morphologic diversity and shared genetics, rather than distinct tumor types with overlapping gene fusions. Of note, PPMS show some histologic overlap and similar genetic features with $\mathrm{AFH}$, despite desmin negativity and the lack of lymphoplasmacytic cuffing, raising the possibility of a pulmonary myxoid variant of AFH.

Kao et al reported of a series of 5 myxoid mesenchymal neoplasms with a remarkable predilection for the meninges and brain in young patients that were characterized by similar morphology and immunoprofile. Their genetic hallmark was the presence of EWSR1 fusions with members of the $C R E B$ gene family (CREM, CREB1, and ATF1) (Table 2).

\section{SMARCB1 (INI1)-Deficient Neoplasia}

SMARCB1, which is also named INI1, is 1 of the core subunit proteins in the SWI/SNF (SWItch/Sucrose Non-Fermentable) ATP-dependent chromatin remodeling complex. SMARCB1/INI1 plays an important role in several pathways related to tumor proliferation and progression (WNT, Sonic hedgehog, p16-RB pathway and Polycomb pathway). The clinicopathologic spectrum of intracranial neoplasms displaying consistent loss of nuclear SMARCB1 (INI1) expression has been steadily expanding to encompass not only the atypical teratoid rhabdoid tumor, but also pediatric chordomas, synovial sarcoma, and the so-called cribriform neuroepithelial tumor (25-27). In our case series, 2 patients (Cases 1 and 2) showed loss of INI1 expression. Whether INI1 deficient intracranial tumors of the type described in this study comprise a separate entity remains to be established.

In the current series, we demonstrated that neoplasms in the clinicopathologic and morphologic spectrum of intracranial EMC are heterogeneous at the molecular level. Notably, none of the 3 cases harbored a NR4A3 gene fusion, highlighting their molecular distinctness from EMC of soft tissues and other extracranial sites. Instead, 1 tumor demonstrated EWSR1-CREB1 gene translocations. The latter aberration has been reported in a variety of mesenchymal and rarely also epithelial neoplasms. In particular, primary pulmonary myxoid sarcoma which is well known to be closely related to EMC morphologically was found to harbor these same EWSR1$C R E B 1$ gene fusions in the vast majority of cases $(18,28)$. Indeed, some reports on EMC primary in the lung have been considered examples of primary pulmonary myxoid sarcoma. In a recent report, Agaimy et al demonstrated loss of SMARCB1 in a case of primary pulmonary myxoid sarcoma that lacked gene fusions in the EWSR1, FUS and NR4A3 gene loci, suggesting SMARCB1 deficiency as an alternative molecular event driving some neoplasms in the morphologic spectrum of primary pulmonary myxoid sarcoma (29). In the current study, we observed same phenomenon where 2 of our 3 cases of intracranial EMC lacked these characteristic gene fusions (both by FISH and NGS) and instead displayed complete loss of SMARCB1 protein by immunohistochemistry as a consequence of monoallelic SMRACBI gene locus deletion demonstrable by FISH.

\section{Conclusion}

Comprehensive analysis of the clinicopathologic features and molecular alterations of intracranial EMC-like neoplasms underscore the genetic variability and molecular heterogeneity of neoplasms in the clinicopathologic spectrum of intracranial EMC. At least in our small series, intracranial EMC seems to be distinctive from conventional (soft tissue) EMC at extracranial sites. Analogous to similar pulmonary lesions, the noncommitted term "primary intracranial myxoid sarcoma" might be more convenient for this rare entity. Molecular suptyping of these rare diseases may be relevant for future management of affected patients and may help to optimize therapeutic decisions.

\section{REFERENCES}

1. Gonzáles-Lois C, Cuevas C, Abdullah O, et al. Intracranial extraskeletal myxoid chondrosarcoma: case report and literature review. Acta Neurochir (Wien) 2002; 144:736-40

2. Hassounah M, Al-Mefty O, Akhtar M, et al. Primary cranial and intracranial chondrosarcoma-A survey. Acta Neurochir (Wien) 1985;78: 123-32

3. Korten AG, ter Berg HJ, Spincemaille GH, et al. Intracranial chondrosarcoma: Review of the literature and report of 15 cases. J Neurol Neurosurg Psychiatry 1998;65:88-92

4. Berkmen YM, Blatt ES. Cranial and intracranial cartilaginous tumours. Clin Radiol 1968; 19:327-33

5. Scott RM, Dickersin GR, Wolpert SM, et al. Myxochondrosarcome of the fourth ventricle. J Neurosurg 1976;44:1-4

6. Chaskis C, Michotte A, Goossens A, et al. Primary intracerebral myxoid chondrosarcoma. J Neurosurg 2002; 97:228

7. Im S-H, Kim DG, Park IA, et al. Primary intracranial myxoid chondrosarcoma. Report of a case and review of the literature. J Korean Med Sci 2003; 18:301-7

8. O'Brien J, Thornton J, Cawley D, et al. Extraskeletal myxoid chondrosarcoma of the cerebellopontine angle presenting during pregnancy. $\mathrm{Br} \mathrm{J}$ Neurosurg 2008;22:429-32

9. Park JH, Kim MJ, Kim CJ, et al. Intracranial extraskeletal myxoid chondrosarcoma: case report and literature review. J Korean Neurosurg Soc 2012;52:246-9

10. Dulou R, Chargari C, Dagain A, et al. Primary intracranial extraskeletal myxoid chondrosarcoma. Neurol Neurochir Pol 2012;46:76-81

11. Lee S, Kim NR, Chung DH, et al. Squash cytology of a dural-based highgrade chondrosarcoma may mimic that of glioblastoma in the central nervous system. Acta Cytol 2015;59:219-24

12. Smith TW, Davidson RI. Primary meningeal myxochondrosarcoma presentig as a cerebellar mass: Case report. Neurosurgery 1981;8:577-81

13. Sorimachi T, Sasaki O, Nakazato S, et al. Myxoid chondrosarcoma in the pineal region. J Neurosurg 2008;109:904-7.

14. Louis DN, Ohgaki H, Wiestler OD, et al. World Health Organization Histological Classification of Tumours of the Central Nervous System. 2016 International Agency for Research on Cancer.

15. De Andrea C, San-Julian M, Bovee J. Integrating morphology and genetics in the diagnosis of cartilage tumors. Surg Pathol Clin 2017;10:537-52

16. Kao Y, Sung Y, Zhang L, et al. EWSR1 fusions with CREB family transcription factors define a novel myxoid mesenchymal tumor with predilection for intracranial location. Am J Surg Pathol 2017;41:482-90 
17. Bale TA, Oviedo A, Kozakewich H, et al. Intracranial myxoid mesenchymal tumors with EWSR1-CREB family gene fusions: myxoid variant of angiomatoid fibrous histiocytoma or novel entity? Brain Pathol 2018;28: 183-91

18. Thway K, Fisher C. Tumors with EWSR1-CREB1 and EWSR1-ATF1 fusions. Am J Surg Pathol 2012;36:e1-11

19. Kohashi K, Oda Y. Oncogenic roles of SMARCB1/INI1 and its deficient tumors. Cancer Sci 2017;108:547-52

20. Agaram NP, Zhang L, Sung YS, et al. Extraskeletal myxoid chondrosarcoma with non-EWSR1-NR4A3 variant fusions correlate with rhabdoid phenotype and high-grade morphology. Hum Pathol 2014;45:1084-91

21. Hisaoka M, Ishida T, Kuo T, et al. Clear cell sarcoma of soft tissue. Am J Surg Pathol 2008;32:452-60

22. Antonescu CR, Tschernyavsky SJ, Woodruff JM, et al. Molecular diagnosis of clear cell sarcoma: Detection of EWS-ATF1 and MITF-M transcripts and histopathological and ultrastructural analysis of 12 cases. J Mol Diagn 2002;4:44-52

23. Antonescu CR, Nafa K, Segal NH, et al. EWS-CREB1: a recurrent variant fusion in clear cell sarcoma-Association with gastrointestinal location and absence of melanocytic differentiation. Clin Cancer Res 2006;12:5356-62
24. Antonescu CR, Dal Cin P, Nafa K, et al. EWSR1-CREB1 is the predominant gene fusion in angiomatoid fibrous histiocytoma. Genes Chromosomes Cancer 2007;46:1051-60

25. Malgulwar PB, Pathak P, Singh M, et al. Downregulation of SMARCB1/ INI1 expression in pediatric chordomas correlates with upregulation of miR-671-5p and miR-193a-5p expressions. Brain Tumor Pathol 2017; 34:155-9

26. Johann PD, Hovestadt V, Thomas C, et al. Cribriform neuroepithelial tumor: Molecular characterization of a SMARCB1-deficient non-rhabdoid tumor with favorable long-term outcome. Brain Pathol 2017;27:411-8

27. Bianchi G, Sambri A, Righi A, et al. Histology and grading are important prognostic factors in synovial sarcoma. Eur J Surg Oncol 2017;43:1733-9

28. Matsukuma S, Hisaoka M, Obara K, et al. Primary pulmonary myxoid sarcoma with EWSR1-CREB1 fusion, resembling extraskeletal myxoid chondrosarcoma: case report with a review of literature. Pathol Int 2012; $62: 817-22$

29. Agaimy A, Duell T, Morresi-Hauf A. EWSR1-fusion-negative, SMARCB1-deficient primary pulmonary myxoid sarcoma. Pol J Pathol 2017;68:261-7

30. Arpino L, Capuano C, Gravina M et al. Parasellar myxoid chondrosarcoma: a rare variant of cranial chondrosarcoma. J Neurosurg. Sci 2011;55:387-89 